\title{
Effect of Ulinastatin Combined with Octreotide on Serum Endothelin, Endotoxin Levels and Immune Function in Acute Pancreatitis
}

\author{
Luyang Meng, Ziheng Wu and Hongkun Zhang
}

\begin{abstract}
The aim of this study was to determine clinical efficacy of ulinastatin combined with octreotide in treatment of acute pancreatitis, and its effect on serum endothelin, endotoxin level and immune function. It was an analytical observational study carried out from September 2016 to March 2018. A total of 108 patients with acute pancreatitis were randomly divided into observation group and control group, 54 cases in each group. Control group was additionally treated with octreotide. Observation group was treated with ulinastatin. Therapeutic effects of two groups were compared. The total effective rate in observation group was higher than that in control group $(p=0.046)$. After seven days of treatment, serum endothelin and endotoxin levels in observation group were lower than those in control group (both $p<0.001$ ); $\operatorname{lgA}$, IgM and IgG levels in observation group were higher than those in control group ( $p=0.031,0.007$, and 0.001 , respectively). Ulinastatin combined with octreotide can reduce level of endothelin and endotoxin and improve immune function.
\end{abstract}

Key Words: Acute pancreatitis, Ulinastatin, Octreotide, Endothelin, Endotoxin, Immune function.

Acute pancreatitis is a common clinical inflammatory reaction in the pancreatic tissue caused by multiple causes, such as self-digestion, edema, hemorrhage or even necrosis. Its clinical features are acute upper abdominal pain, fever, nausea, vomiting, and elevated blood coagulase levels.1,2 Patients with acute pancreatitis have an acute illness and rapid progress. If they are not treated in time, it may even endanger the lives of patients.

The treatment of acute pancreatitis is mainly on ulinastatin and octreotide administration. Presence of immunosuppressive reactions in the early stages of patients with acute pancreatitis may seriously affect the prognosis of the disease. ${ }^{3}$ Endothelin and endotoxin are involved in the occurrence and development of acute pancreatitis. ${ }^{4}$ Elevated endothelin on the one hand will promote the pancreatic microcirculation disorder; on the other hand, will cause mesenteric vasoconstriction, resulting in gastrointestinal ischemia, mucosal damage, increase endotoxemia. At present, there are few studies on the effects of ulinastatin combined with octreotide on serum endothelin, endotoxin levels and immune function in patients with acute pancreatitis.

Department of Vascular Surgery, The First Affiliated Hospital, School of Medicine, Zhejiang University,Hangzhou City,

Zhejiang Province, 310009, China

Correspondence: Dr. Hongkun Zhang, Department of Vascular Surgery, The First Affiliated Hospital, School of Medicine,

Zhejiang University, Hangzhou City, Zhejiang Province,

310009, China

E-mail:rzwi69@163.com

Received: May 23, 2018; Accepted: September 28, 2018
The objective of this study was to investigate the clinical efficacy of ulinastatin combined with octreotide in the treatment of acute pancreatitis, and to analyse its effect on serum endothelin, endotoxin levels and immune function.

This observational study was conducted at Department of Vascular Surgery, The First Affiliated Hospital, School of Medicine, Zhejiang University, China, from September 2016 to March 2018. It was approved by the Hospital Ethics Committee, and all patients volunteered to participate in the study. Inclusion criteria were the condition of acute onset with continuous abdominal pain, abdominal muscle tension, nausea and vomiting, elevated serum amylase level, higher than normal; confirmed acute pancreatitis according to physical signs, symptoms, laboratory and imaging examinations course $<5$ days. Exclusion criteria were pregnant and lactating women; history of intestinal disease, recent use of hormones, enzymic preparations, allergic constitution, patients with severe hepatic and gallbladder, renal and cardio-cerebral diseases. The patients were randomly divided into observation group and control group by using random number table method, with 54 cases in each group.

All patients received routine surgical treatment after admission, including abrosia, continuous monitoring of vital signs, gastrointestinal decompression, antibiotic prevention or control of infection, spasmolysis and analgesia, proton pump acid suppression, blood volume supplementation, fluid infusion, and regulation of disturbance of water and electrolyte, correction of acid-base balance, improvement of microcirculation, nutrition support, etc. The control group was treated 
Table I: Comparison of clinical efficacy between the two groups.

\begin{tabular}{l|c|c|c|c|c}
\hline Groups & $\mathrm{n}$ & $\begin{array}{c}\text { Excellent } \\
\mathrm{n}(\%)\end{array}$ & $\begin{array}{c}\text { Valid } \\
\mathrm{n}(\%)\end{array}$ & $\begin{array}{c}\text { Invalid } \\
\mathrm{n}(\%)\end{array}$ & $\begin{array}{c}\text { Total effective } \\
\mathrm{n}(\%)\end{array}$ \\
\hline Control group & 54 & $15(27.78)$ & $31(57.41)$ & $8(14.81)$ \\
Observation group & 54 & $23(42.59)$ & $29(53.70)$ & $46(85.19)$ \\
\hline
\end{tabular}

Table II: Comparison of serum endothelin, endotoxin and immune function indexes after 7 days of treatment in both groups.

\begin{tabular}{|c|c|c|c|c|c|c|c|c|c|c|c|}
\hline \multirow[t]{2}{*}{ Groups } & \multirow[t]{2}{*}{$\mathrm{n}$} & \multicolumn{2}{|c|}{ Endothelin (ng/L) } & \multicolumn{2}{|c|}{ Endotoxin $(\mathrm{EU} / \mathrm{mL})$} & \multicolumn{2}{|c|}{$\lg A(g / L)$} & \multicolumn{2}{|c|}{$\operatorname{lgM}(g / L)$} & \multicolumn{2}{|c|}{$\lg G(g / L)$} \\
\hline & & Mean $\pm S D$ & $p$-value & Mean $\pm S D$ & $p$-value & Mean \pm SD & $\mathrm{p}$-value & Mean \pm SD & $\mathrm{p}$-value & Mean \pm SD & $p$-value \\
\hline Contr & 54 & $73.26 \pm 12.29$ & $<0.001$ & $0.34 \pm 0.06$ & $<0.001$ & $4.33 \pm 1.41$ & 0.031 & $1.48 \pm 0.49$ & 0.007 & $10.28 \pm 1.84$ & 0.001 \\
\hline Observation group & 54 & $55.48 \pm 8.74$ & & $0.16 \pm 0.03$ & & $4.96 \pm 1.59$ & & $1.75 \pm 0.52$ & & $11.41 \pm 1.56$ & \\
\hline
\end{tabular}

with a microvenous pump used for continuous intravenous pumping of $500 \mathrm{~mL}$ physiological saline $+0.6 \mathrm{mg}$ octreotide once a day for seven days at a rate of $25 \mathrm{~g} / \mathrm{h}$. The observation group was additionally treated with intravenous drip of $250 \mathrm{~mL} 5 \%$ glucose injection $+100,000 \mathrm{U}$ ulinastatin injection was completed within two hours, twice a day, for seven days. Efficacy and related indicators were evaluated after seven days of treatment in both groups.

After seven days of treatment, the clinical efficacy of the two groups of patients was evaluated. Excellent was labelled if the symptoms and signs such as nausea, vomiting, abdominal distension and abdominal pain disappeared, and the urinary amylase has been reduced to the normal range or the reduction is more than $3 / 4$. Effective was labelled when the relevant clinical symptoms and signs markedly improved and the urinary amylase reduced by more than $1 / 2$. It was non-effective when there was no remarkable changes in the relevant clinical symptoms and signs, or even worsening; urinary amylase did not markedly reduced or complications devoloped. Total effective rate of treatment was (Excellent + Effective) case number / total case number $\times 100 \%$. Simultaneously, after seven days of treatment, $5 \mathrm{~mL}$ of peripheral venous blood was collected from the two groups of patients. The blood was centrifuged to collect serum. Serum endothelin levels were determined by radioimmunoassay. Limulus ameobatic chromogenic assay was used to determine serum endotoxin. Immunoturbidimetry was used to determine the changes of immunoglobulin ( $\lg A, \lg M, \lg G)$ and other immune function indicators.

SPSS version 25.0 software was adopted for data statistical analysis. Measurement data was shown in mean $\pm S D$, examined by independent sample t-test. Enumeration data was represented by $\mathrm{n}(\%)$, and $\mathrm{X}^{2}$ test was adopted to examine them. The $p<0.05$ means there was statistical significance in the difference.

Among the 108 patients, there were 59 (54.63\%) males and $49(45.37 \%)$ females, aged $34-56$ years, with average age of $48.61 \pm 3.27$ years. The course of disease was 1-4 days and the average duration of disease was $1.35 \pm 0.27$ days. The total effective rate in the observation group was $96.30 \%$ (52 cases), which was higher than that in the control group $85.19 \%$ (46 cases) $(p=0.046$, Table I). After seven days of treatment, serum endothelin and endotoxin levels in the observation group were lower than those in the control group (both $p<0.001$ ); $\lg \mathrm{A}, \lg \mathrm{M}$ and $\lg \mathrm{G}$ levels in the observation group were higher than those in the control group $(p=0.031,0.007$, and 0.001 , respectively, Table II).

For the past few years, octreotide and ulinastatin have received increasing attention in the treatment of acute pancreatitis. Octreotide is an octapeptide cyclic compound, which acts similar to natural endogenous somatostatin and can effectively control and regulate the secretion of pancreatic enzyme, cholecystokinin, pancreatic juice, etc., slow down the movement and emptying of the stomach and the gallbladder to inhibit the emptying of the bile and produce protective effects on the pancreatic parenchymal cells. Ulinastatin is a broad-spectrum protease inhibitor widely used clinically. It is a trypsin inhibitor, which is widespread in human urine. It not only inhibits sugars and lipids, but also inhibits various proteases, can effectively prevent the excessive release of inflammatory mediators. By means of inhibiting the release of tumor necrosis factor, it can reduce the self digestion of enzymes, regulate the function of vascular endothelial cells, reduce self-injury, improve microcirculation and the absorption of endotoxin, stabilise lysozyme and inhibit lysosome release. The results of this study showed that the total effective rate of treatment in the observation group was higher than that of the control group. This suggests that the clinical efficacy of ulinastatin combined with octreotide in the treatment of acute pancreatitis is superior to that of octreotide alone. The reason may be that the combination of ulinastatin and octreotide can synergistically improve the function of pancreas.

Studies have shown that intestinal endotoxin can promote the conversion of acute edematous pancreatitis to acute necrotising pancreatitis. ${ }^{5}$ The authors clinically observed significant increases in plasma endothelin and endotoxin in patients with acute pancreatitis. The mechanism may be a severely damaged intestinal mucosal barrier during acute necrotising pancreatitis, intestinal endotoxin increases to vascular endothelial cells, and the damaged vascular endothelial cells 
increasingly express and release endothelin, which results in pancreas microcirculation disturbances, leading to severe pancreatic damage. The results of this study showed that combination therapy of ulinastatin and octreotide can reduce the serum endothelin and endotoxin levels in patients with acute pancreatitis, and reduce the clinical symptoms of patients.

Measurement of serum immunoglobulin $(\mathrm{lg})$ is the most commonly used method for checking humoral immunity function. Usually, serum Ig levels are expressed as IgA, IgM, IgG. When acute pancreatitis occurs, the immune function in the body is often disordered, and the levels of $\lg A, \lg M$, and $\lg G$ are reduced. The results of this study showed that the combination of ulinastatin and octreotide can improve the level of $\lg \mathrm{A}$, $\lg \mathrm{M}$ and $\lg \mathrm{g}$ in patients. The reason may be that, as a somatostatin, octreotide has a certain degree of immunomodulatory effect, and can also enhance the effect of ulinastatin on immune function, showing a superimposed effect.

This study shows that ulinastatin combined with octreotide has significant effect on acute pancreatitis, which can reduce the levels of endothelin and endotoxin in patients, and improve the immune function of patients. It has certain clinical application value, too.

\section{REFERENCES}

1. Losurdo G, lannone A, Principi M, Barone M, Ranaldo N, lerardi $\mathrm{E}$, et al. Acute pancreatitis in elderly patients: a retrospective evaluation at hospital admission. Eur $J$ Intern Med 2016; 30:88-93.

2. Topaloglu N, Kucuk A, Tekin M, Yildirim S, Erbas M, Kiraz HA, et al. Serum ischemia-modified albumin levels in experimental model of acute pancreatitis. J Coll Physicians Surg Pak 2015; 25:395-8.

3. Crockett Wani S, Gardner TB, Falck-Ytter Y, Barkun AN. American gastroenterological association institute guideline oninitial management of acute pancreatitis. Gastroenterology 2018; 154:1096-101

4. Mifkovic A, Skultety J, Pindak D, Pechan J. Specific aspects of acute pancreatitis. Bratisl Lek Listy 2009; 110:544-52.

5. Wang X, Wu L, Wu K, Zhang R, Dong Y. Roles of endotoxinrelated signaling molecules in the progression of acute necrotizing pancreatitis in mice. Pancreas 2005; 31: 251-7. 